Supplement of Atmos. Chem. Phys., 20, 13611-13626, 2020

https://doi.org/10.5194/acp-20-13611-2020-supplement

(C) Author(s) 2020. This work is distributed under

the Creative Commons Attribution 4.0 License.

(c) (1)

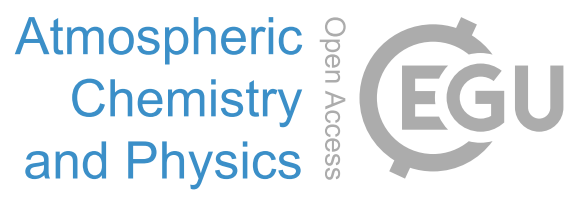

Supplement of

\title{
On mineral dust aerosol hygroscopicity
}

Lanxiadi Chen et al.

Correspondence to: Mingjin Tang (mingjintang@gig.ac.cn)

The copyright of individual parts of the supplement might differ from the CC BY 4.0 License. 


\section{Table of contents}

2

3

4 Work.

5 6 work.

7

8

9

10

Table S1: Mass fraction of soluble cations for the mineral dust samples investigated in this

Table S2: Mass fraction of soluble anions for the mineral dust samples investigated in this

Figures S1-S7: Particle size distribution of mineral dust samples examined in this work. 
11 Table S1. Mass fraction (mg per $\mathrm{g}$ ) of soluble cations for the mineral dust samples investigated in

12 this work (n. d.: not detected).

\begin{tabular}{|c|c|c|c|c|c|c|}
\hline sample & $\mathrm{Na}^{+}$ & $\mathrm{NH}_{4}^{+}$ & $\mathrm{K}^{+}$ & $\mathrm{Ca}^{2+}$ & $\mathrm{Mg}^{2+}$ & cations \\
\hline $\mathrm{SiO}_{2}$ & n. d. & n. d. & 0.04 & n. d. & 0.06 & 0.11 \\
\hline $\mathrm{TiO}_{2}$ & n. d. & n. d. & n. d. & n. d. & n. d. & n. d. \\
\hline hematite & 0.36 & 0.32 & n. d. & n. d. & n. d. & 0.68 \\
\hline goethite & 0.08 & n. d. & 0.07 & 0.63 & 0.91 & 1.69 \\
\hline magnetite & 0.15 & n. d. & n. d. & n. d. & n. d. & 0.15 \\
\hline potassium feldspar & 0.30 & n. d. & 0.79 & 0.06 & 0.58 & 1.73 \\
\hline albite & 1.48 & n. d. & n. d. & n. d. & n. d. & 1.48 \\
\hline microcline & 0.24 & n. d. & 1.03 & 0.29 & 0.47 & 2.02 \\
\hline $\mathrm{CaCO}_{3}$ & n. d. & n. d. & n. d. & n. d. & 6.73 & 6.73 \\
\hline dolomite & 0.01 & n. d. & n. d. & 3.03 & 8.81 & 11.85 \\
\hline illite & 0.08 & n. d. & 0.72 & 0.29 & 0.80 & 1.89 \\
\hline kaolinite & 0.01 & n. d. & 0.08 & n. d. & 0.02 & 0.10 \\
\hline montmorillonite & 0.06 & n. d. & 0.05 & n. d. & 0.37 & 0.48 \\
\hline chlorite & n. d. & n. d. & 0.05 & 0.47 & 0.08 & 0.60 \\
\hline ATD & 1.01 & n. d. & 1.20 & 0.38 & 6.10 & 8.69 \\
\hline China loess & 0.35 & n. d. & 0.39 & 0.27 & 3.47 & 4.47 \\
\hline QH dust & 0.64 & n. d. & 0.51 & 0.38 & 3.97 & 5.51 \\
\hline TLF dust & 0.78 & 0.02 & 0.49 & 0.32 & 6.46 & 8.07 \\
\hline Bordj dust & 1.60 & n. d. & 0.29 & 0.09 & 3.58 & 5.56 \\
\hline M'Bour dust & n. d. & n. d. & 0.18 & 0.09 & 0.62 & 0.89 \\
\hline Saharan dust & 2.90 & n. d. & 0.52 & 0.21 & 0.31 & 3.93 \\
\hline
\end{tabular}


16 Table S2. Mass fraction (mg per $\mathrm{g}$ ) of soluble anions for the mineral dust samples investigated in 17 this work (n. d.: not detected).

\begin{tabular}{|c|c|c|c|c|c|c|c|}
\hline sample & $\mathrm{F}^{-}$ & $\mathrm{Cl}^{-}$ & $\mathrm{NO}_{3}^{-}$ & $\mathrm{SO}_{4}{ }^{2-}$ & $\mathrm{PO}_{4}^{3-}$ & anions * & total $^{\#}$ \\
\hline $\mathrm{SiO}_{2}$ & n. d. & 0.06 & n. d. & n. d. & n. d. & 0.06 & 0.16 \\
\hline $\mathrm{TiO}_{2}$ & 0.10 & 1.26 & n. d. & n. d. & n. d. & 1.37 & 1.37 \\
\hline hematite & 0.10 & 0.06 & 0.08 & 2.37 & n. d. & 2.62 & 3.30 \\
\hline goethite & 0.11 & 0.08 & 0.07 & 2.74 & n. d. & 3.00 & 4.69 \\
\hline magnetite & n. d. & 0.08 & n. d. & 0.41 & n. d. & 0.49 & 0.64 \\
\hline $\begin{array}{l}\text { potassium } \\
\text { feldspar }\end{array}$ & 0.05 & 0.11 & 0.02 & 0.08 & n. d. & 0.27 & 2.00 \\
\hline albite & 0.10 & 0.13 & 0.01 & n. d. & n. d. & 0.24 & 1.72 \\
\hline microcline & n. d. & 0.38 & n. d. & n. d. & n. d. & 0.38 & 2.39 \\
\hline $\mathrm{CaCO}_{3}$ & n. d. & 0.07 & n. d. & n. d. & n. d. & 0.07 & 6.80 \\
\hline dolomite & 0.10 & 0.17 & n. d. & n. d. & n. d. & 0.27 & 12.12 \\
\hline illite & 0.09 & 0.09 & n. d. & 0.84 & n. d. & 1.03 & 2.91 \\
\hline kaolinite & 0.06 & 0.08 & n. d. & 0.21 & n. d. & 0.35 & 0.45 \\
\hline montmorillonite & 0.11 & 0.21 & n. d. & 0.30 & n. d. & 0.61 & 1.09 \\
\hline chlorite & 0.11 & 0.06 & n. d. & n. d. & n. d. & 0.17 & 0.77 \\
\hline ATD & 0.11 & 1.35 & 0.08 & 0.62 & n. d. & 2.15 & 10.84 \\
\hline China loess & n. d. & n. d. & n. d. & n. d. & n. d. & n. d. & 4.47 \\
\hline QH dust & n. d. & 0.16 & n. d. & 2.07 & n. d. & 2.23 & 7.74 \\
\hline TLF dust & 0.10 & 0.51 & 0.13 & 0.94 & n. d. & 1.68 & 9.75 \\
\hline Bordj dust & 0.05 & 1.72 & 0.09 & 5.12 & n. d. & 6.98 & 12.55 \\
\hline M'Bour dust & n. d. & 0.11 & 0.31 & 0.13 & n. d. & 0.55 & 1.44 \\
\hline Saharan dust & n. d. & 3.99 & 0.06 & 0.97 & 0.95 & 5.97 & 9.90 \\
\hline
\end{tabular}

18 *: Total mass fractions of all the anions; total mass fractions of all the ions.

19 

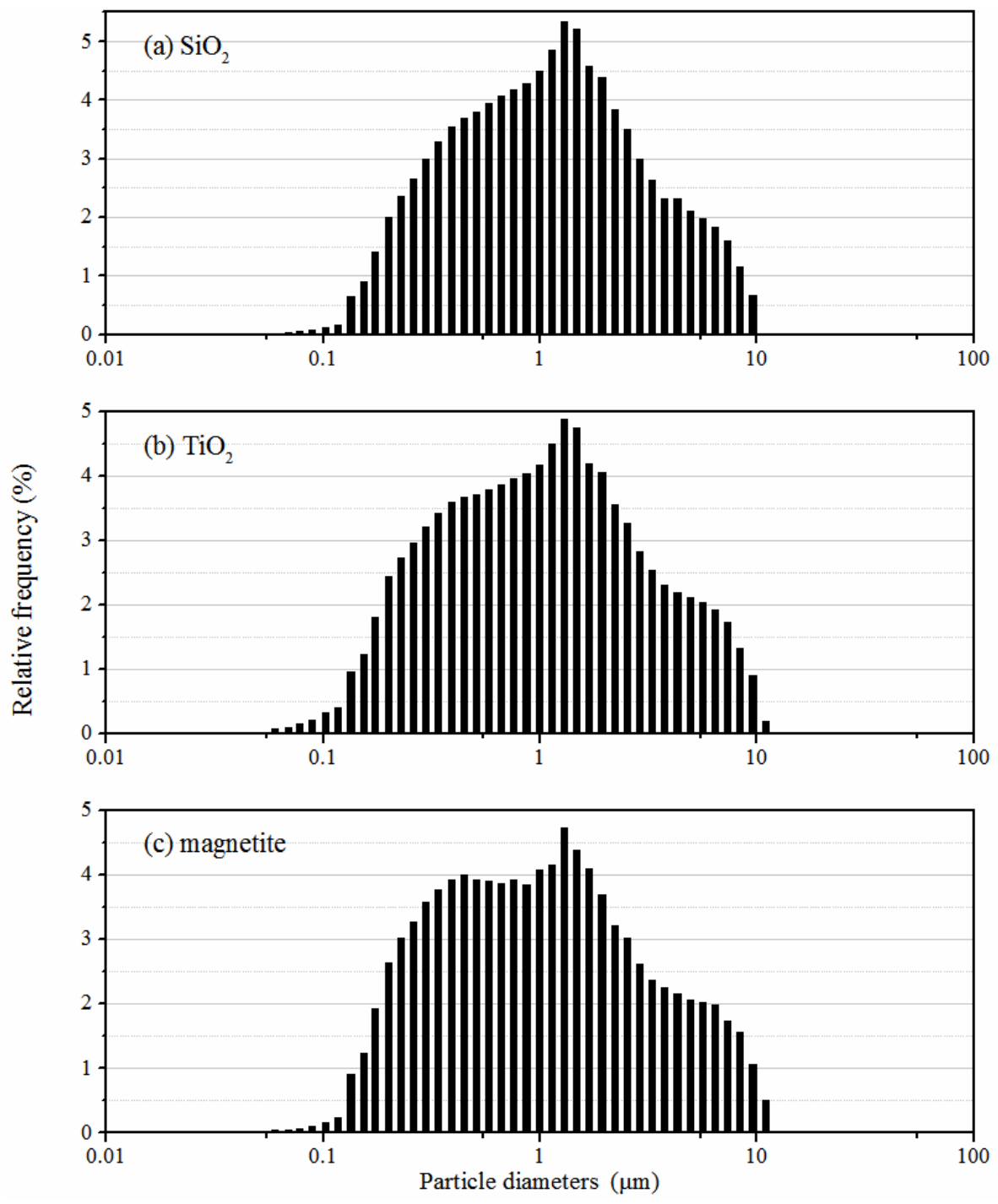

23 Figure S1. Particle size distribution of (a) $\mathrm{SiO}_{2}$, (b) $\mathrm{TiO}_{2}$ and (c) magnetite.

24

25 

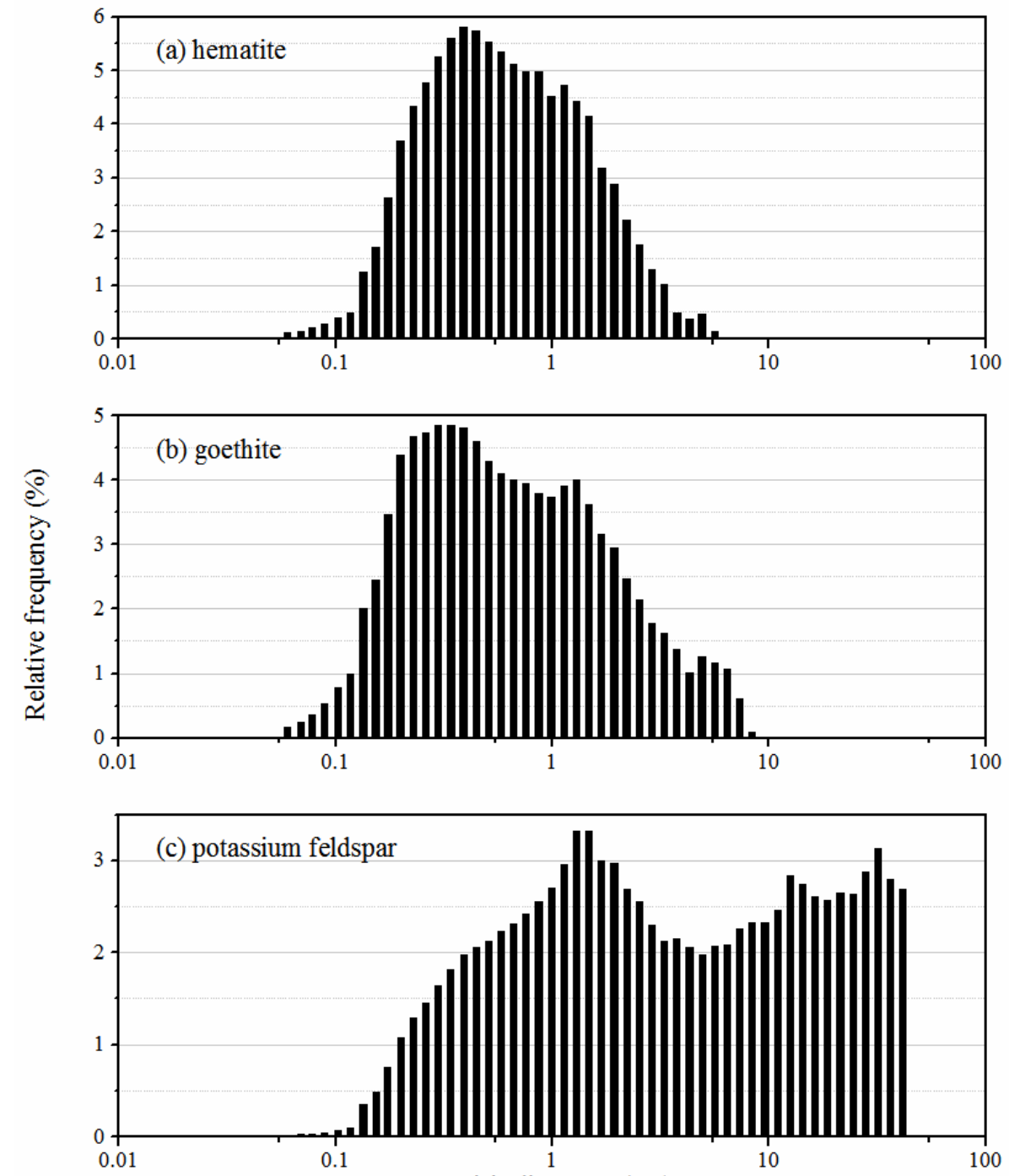

27 Figure S2. Particle size distribution of (a) hematite, (b) goethite and (c) potassium feldspar. 

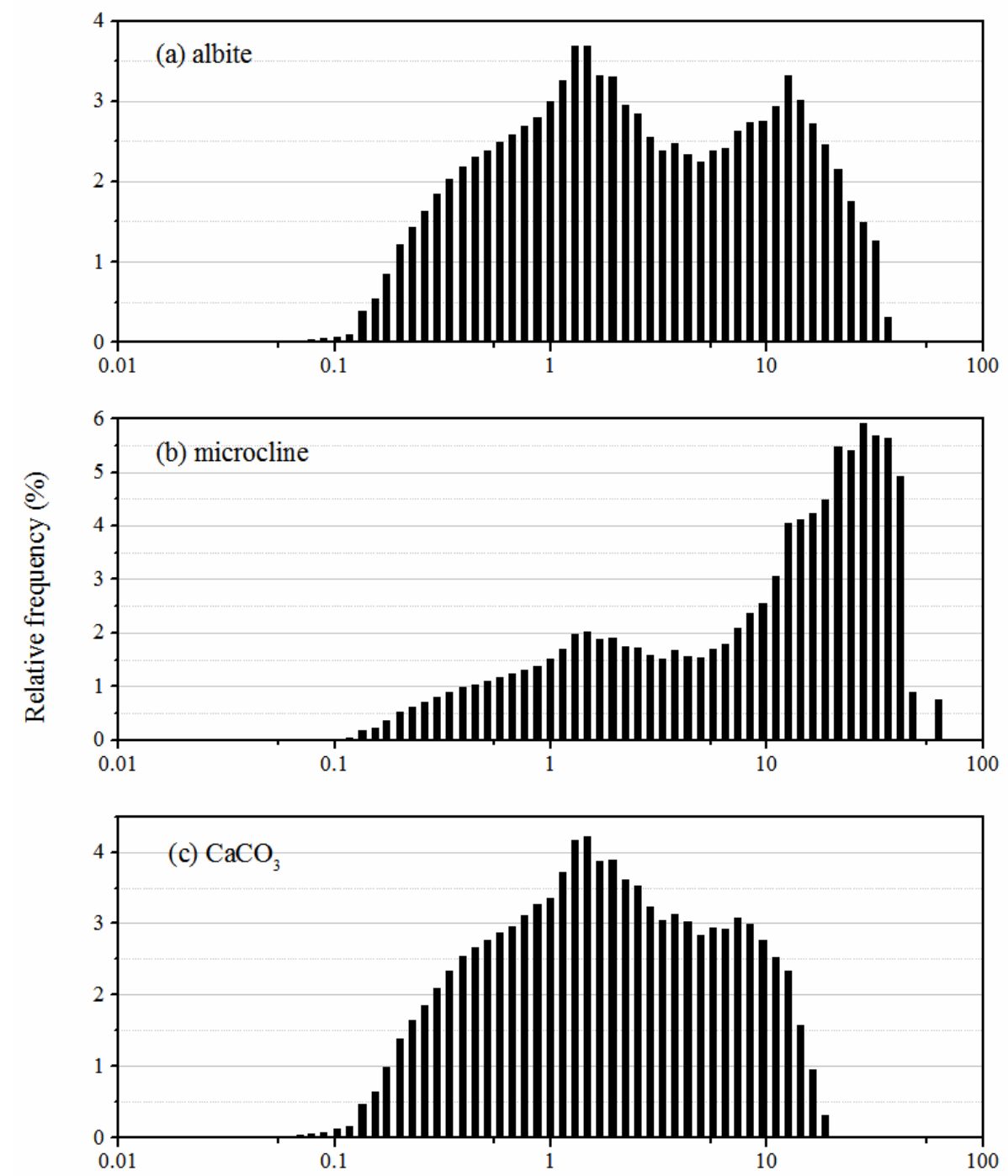

31 Figure S3. Particle size distribution of (a) albite, (b) microcline and (c) $\mathrm{CaCO}_{3}$. 

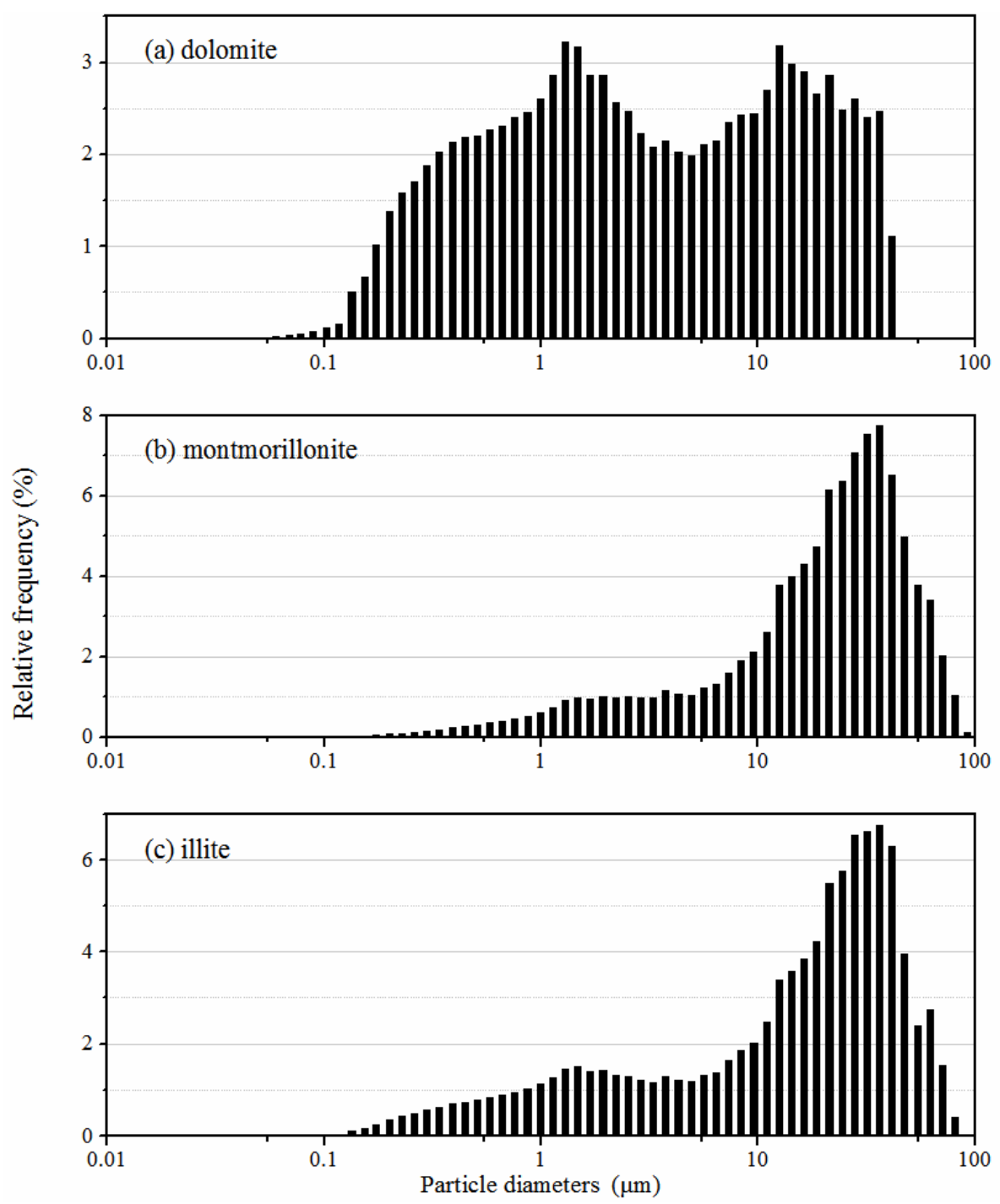

35 Figure S4. Particle size distribution of (a) dolomite, (b) montmorillonite and (c) illite.

36

37 

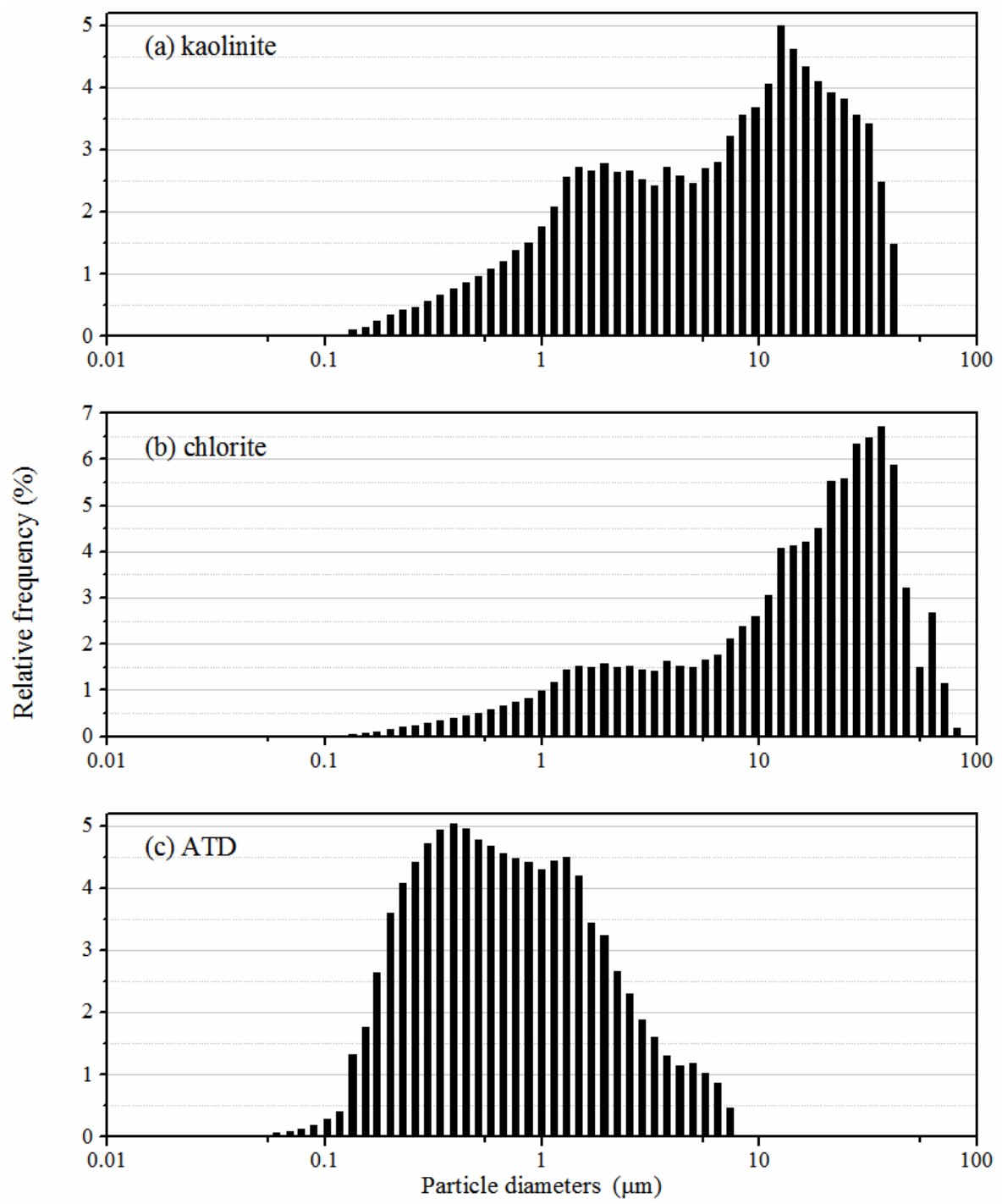

39 Figure S5. Particle size distribution of (a) kaolinite, (b) chlorite and (c) ATD. 

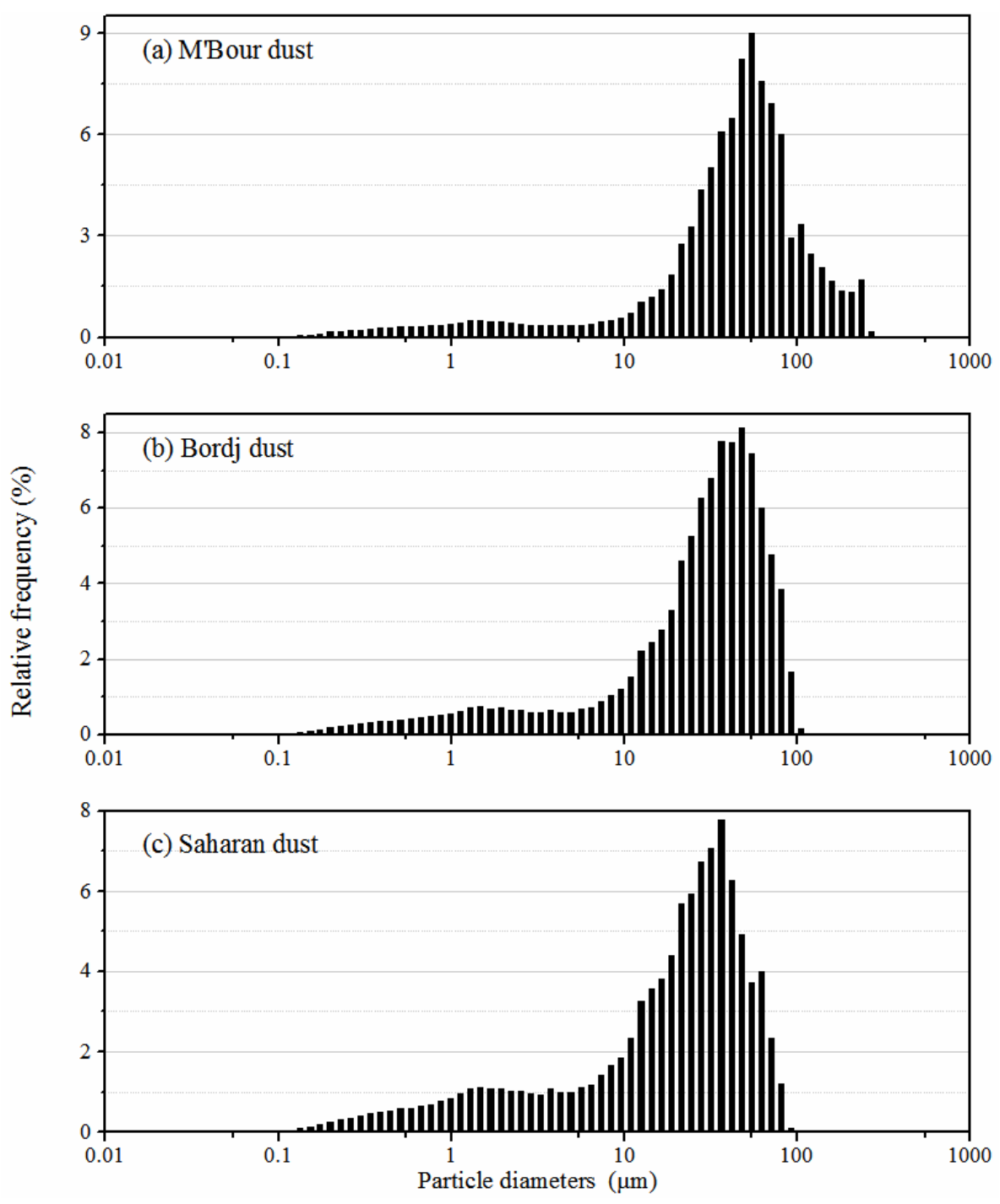

43 Figure S6. Particle size distribution of (a) M'Bour dust, (b) Bordj dust and (c) Saharan dust. 

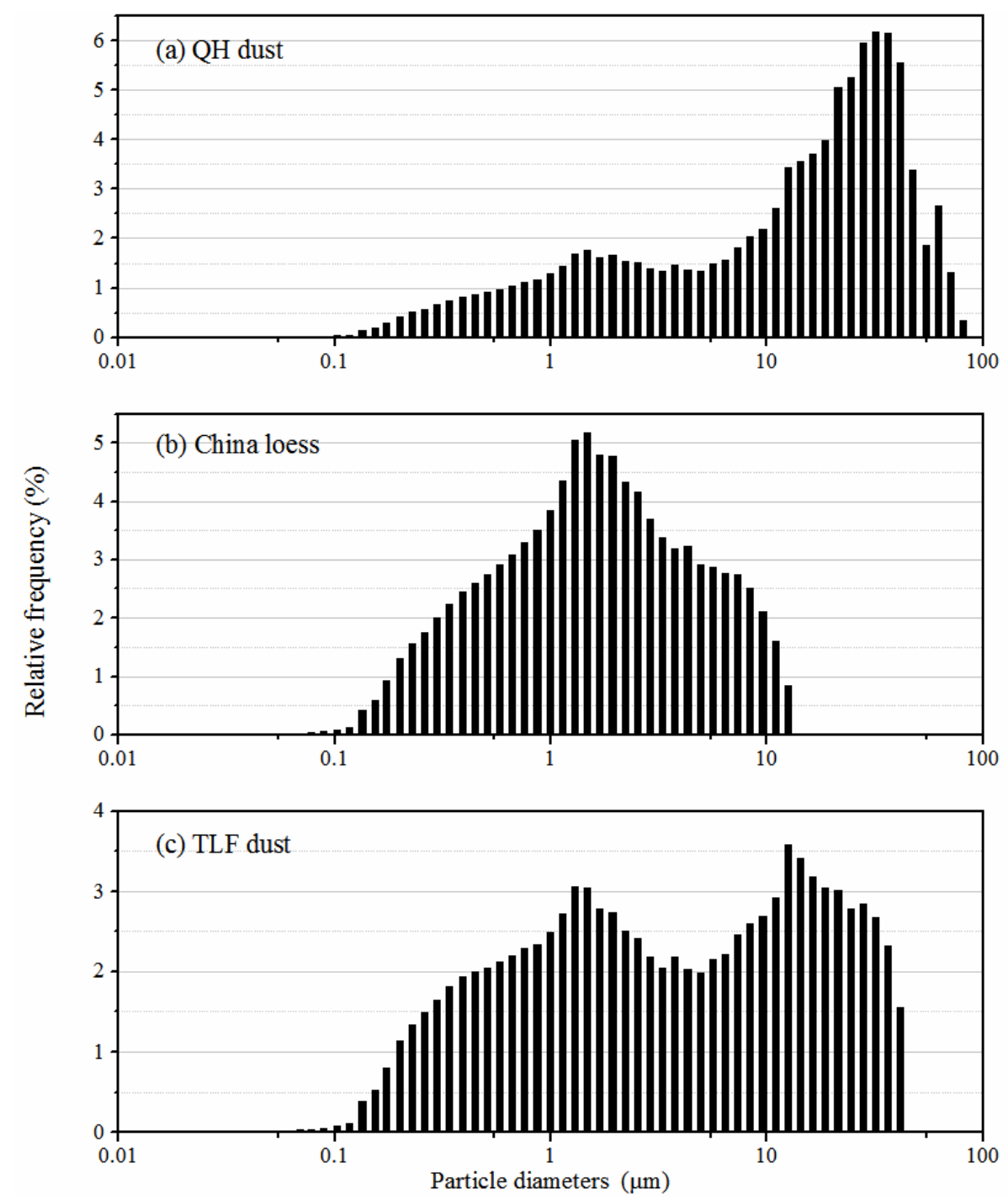

47 Figure S7. Particle size distribution of (a) QH dust, (b) China loess and (c) TLF dust.

48

49

50 